\author{
B.T. Kalimbetov ${ }^{1}$, V.F. Safonov ${ }^{2}$ \\ ${ }^{1}$ H.A. Yasawi International kazakh-turkish University, Turkestan, Kazakhstan; \\ ${ }^{2}$ National Research University «Moscow Power Engineering Institute», Russia \\ (E-mail: burkhan.kalimbetov@ayu.edu.kz,SafonovVF@yandex.ru)
}

\title{
Integro-differentiated singularly perturbed equations with fast oscillating coefficients
}

\begin{abstract}
In the study of various issues related to dynamic stability, with the properties of media with a periodic structure, in the study of other applied problems, one has to deal with differential equations with rapidly oscillating coefficients. Asymptotic integration of differential systems of equations with such coefficients was carried out by the splitting method and the regularization method. In this paper, a system of integrodifferential equations is considered. The main objective of the study is to identify the influence of the integral term on the asymptotics of the solution to the original problem. The case of the absence of resonance is considered, i.e. the case when the integer linear combination of frequencies of the rapidly oscillating coefficient does not coincide with the frequency of the spectrum of the limit operator.
\end{abstract}

Keywords: singularly perturbation, integro-differential equation, rapidly oscillating coefficient, regularization, asymptotic convergence.

\section{Introduction}

Consider the following integro-differential system:

$$
\varepsilon \frac{d z}{d t}-A(t) z-\varepsilon g(t) \cos \frac{\beta(t)}{\varepsilon} B(t) z-\int_{t_{0}}^{t} K(t, s) z(s, \varepsilon) d s=h(t), \quad z\left(t_{0}, \varepsilon\right)=z^{0}, \quad t \in\left[t_{0}, T\right],
$$

where $z=\left\{z_{1}, z_{2}\right\}, h(t)=\left\{h_{1}(t), h_{2}(t)\right\}, \beta^{\prime}(t)>0, \omega(t)>0\left(\forall t \in\left[t_{0}, T\right]\right), g(t)$ is a scalar function, $A(t)$ and $B(t)$ are $(2 \times 2)$ - matrices, with $A(t)=\left(\begin{array}{rr}0 & 1 \\ -\omega^{2}(t) & 0\end{array}\right), z^{0}=\left\{z_{1}^{0}, z_{2}^{0}\right\}, \varepsilon>0$ is a small parameter. Such a system in the case $\beta(t)=2 \gamma(t), B(t)=\left(\begin{array}{ll}0 & 0 \\ 1 & 0\end{array}\right)$ of the absence of an integral term was considered in [1-6]. In the present work, ideas of the regularization method [3-6] are generated on singularly perturbed systems of integro-differential equations with rapidly oscillating coefficients. The study of singularly perturbed integrodifferential problems by the regularization method of S.A. Lomov [3,4] with unstable values of the kernel of an integral operator is reflected in [7-12]. It should also be noted that it is the merit of V.F. Safonov and A.A. Bobodzhanov in the development of the theory of singularly perturbed integro-differential equations [13-16]. In their studies, various problems for integro-differential systems were considered: with diagonal kernel degenerations, with inverse time, with rapidly changing kernels, with rapidly varying kernels, with partial derivatives, etc. [17-21].

In the system the limiting operator $A(t)$ has a spectrum $\lambda_{1}(t)=-i \omega(t), \quad \lambda_{2}(t)=+i \omega(t), \quad \beta^{\prime}(t) \quad$ is a frequency of rapidly oscillating cosine. In the following, functions $\lambda_{3}(t)=-i \beta^{\prime}(t), \lambda_{4}(t)=+i \beta^{\prime}(t)$ will be called the spectrum of a rapidly oscillating coefficient.

We assume that the following conditions are fulfilled:

1) $\omega(t), \beta(t), g(t) \in C^{\infty}\left(\left[t_{0}, T\right], \mathbb{C}^{1}\right), h(t) \in C^{\infty}\left(\left[t_{0}, T\right], \mathbb{C}^{2}\right)$,

$B(t) \in C^{\infty}\left(\left[t_{0}, T\right], \mathbb{C}^{2 \times 2}\right), K(t, s) \in C^{\infty}\left(\left[t_{0}, T\right], \mathbb{C}^{2 \times 2}\right)$,

2) for $\forall t \in\left[t_{0}, T\right]$ and $n_{3} \neq n_{4}$ inequalities

$$
\begin{aligned}
& n_{3} \lambda_{3}(t)+n_{4} \lambda_{4}(t) \neq \lambda_{j}(t), \\
& \lambda_{k}(t)+n_{3} \lambda_{3}(t)+n_{4} \lambda_{4}(t) \neq \lambda_{j}(t), k \neq j, k, j=1,2,
\end{aligned}
$$

for all multi-indices $n=\left(n_{3}, n_{4}\right)$ with $|n| \equiv n_{3}+n_{4} \geq 1\left(n_{3}\right.$ and $n_{4}$ are non-negative integers) are holds.

We will develop an algorithm for constructing a regularized [3] asymptotic solution of problem (1). Condition 2 ) is called the absence of resonance condition. 


\section{Regularization of problem (1)}

Denote by $\sigma_{j}=\sigma_{j}(\varepsilon)$, independent of $t$ magnitudes $\sigma_{1}=e^{-\frac{i}{\varepsilon} \beta\left(t_{0}\right)}, \sigma_{1}=e^{+\frac{i}{\varepsilon} \beta\left(t_{0}\right)}$, and rewrite system (1) as

$$
\begin{gathered}
\varepsilon \frac{d z}{d t}-A(t) z-\varepsilon \frac{g(t)}{2}\left(e^{-\frac{i}{\varepsilon} \int_{t_{0}}^{t} \beta^{\prime}(\theta) d \theta} \sigma_{1}+e^{+\frac{i}{\varepsilon} \int_{t_{0}}^{t} \beta^{\prime}(\theta) d \theta} \sigma_{2}\right) B(t) z- \\
-\int_{t_{0}}^{t} K(t, s) z(s, \varepsilon) d s=h(t), z\left(t_{0}, \varepsilon\right)=z^{0}, t \in\left[t_{0}, T\right] .
\end{gathered}
$$

We introduce regularizing variables $[3,4]$

$$
\tau_{j}=\frac{1}{\varepsilon} \int_{t_{0}}^{t} \lambda_{j}(\theta) d \theta \equiv \frac{\psi_{j}(t)}{\varepsilon}, j=\overline{1,4}
$$

and instead of problem (2), consider the problem

$$
\begin{gathered}
\varepsilon \frac{\partial \tilde{z}}{\partial t}+\sum_{j=1}^{4} \lambda_{j}(t) \frac{\partial \tilde{z}}{\partial \tau_{j}}-A(t) \tilde{z}-\varepsilon \frac{g(t)}{2}\left(e^{\tau_{3}} \sigma_{1}+e^{\tau_{4}} \sigma_{2}\right) B(t) \tilde{z}- \\
-\int_{t_{0}}^{t} K(t, s) \tilde{z}\left(s, \frac{\psi(s)}{\varepsilon}, \varepsilon\right) d s=h(t),\left.\quad \tilde{z}(t, \tau, \varepsilon)\right|_{t=t_{0}, \tau=0}=z^{0}, t \in\left[t_{0}, T\right],
\end{gathered}
$$

for the function $\tilde{z}=\tilde{z}(t, \tau, \varepsilon)$, where is indicated (by $(3)): \tau=\left(\tau_{1}, \tau_{2}, \tau_{3}, \tau_{4}\right), \psi=\left(\psi_{1}, \psi_{2}, \psi_{3}, \psi_{4}\right)$. It is clear that if $\tilde{z}=\tilde{z}(t, \tau, \varepsilon)$ is a solution to problem (4), then the vector function $z=\tilde{z}\left(t, \frac{\psi(t)}{\varepsilon}, \varepsilon\right)$ is an exact solution to problem (2), therefore, problem (4) is extended with respect to problem (2). However, it cannot be considered fully regularized, since it does not regularize the integral term $J \tilde{z}=\int_{t_{0}}^{t} K(t, s) \tilde{z}\left(s, \frac{\psi(s)}{\varepsilon}, \varepsilon\right) d s$. To regularize the integral operator, we introduce a class $M_{\varepsilon}$ that is asymptotically invariant with respect to the operator $J \tilde{z}[3 ; 62]$. Recall the corresponding concept.

Definition 1. A class $M_{\varepsilon}$ is said to be asymptotically invariant (with $\varepsilon \rightarrow+0$ ) with respect to an operator $P_{0}$ if the following conditions are fulfilled:

1) $M_{\varepsilon} \subset D\left(P_{0}\right)$ with each fixed $\varepsilon>0$;

2) the image $P_{0} g(t, \varepsilon)$ of any element $g(t, \varepsilon) \in M_{\varepsilon}$ decomposes in a power series

$$
P_{0} g(t, \varepsilon)=\sum_{n=0}^{\infty} \varepsilon^{n} g_{n}(t, \varepsilon)\left(\varepsilon \rightarrow+0, g_{n}(t, \varepsilon) \in M_{\varepsilon}, n=0,1, \ldots\right),
$$

convergent asymptotically for $\varepsilon \rightarrow+0$ (uniformly with $t \in\left[t_{0}, T\right]$ ).

From this definition it can be seen that the class $M_{\varepsilon}$ depends on the space $U$, in which the operator $P_{0}$ is defined. In our case $P_{0}=J$. For the space $U$ we take the space of vector functions $z(t, \tau)$, represented by sums

$$
\begin{gathered}
z(t, \tau, \sigma)=z_{0}(t, \sigma)+\sum_{i=1}^{4} z_{i}(t, \sigma) e^{\tau_{i}}+\sum_{2 \leq|m| \leq N_{z}}^{*} z^{m}(t, \sigma) e^{(m, \tau)}+\sum_{j=1}^{2} \sum_{1 \leq|m| \leq N_{z}}^{*} z^{e_{j}+m}(t, \sigma) e^{\left(e_{j}+m, \tau\right)} \\
m=\left(0,0, m_{3}, m_{4}\right), z_{i}(t, \sigma), z^{m}(t, \sigma), z^{e_{j}+m}(t, \sigma) \in C^{\infty}\left(\left[t_{0}, T\right], \mathbb{C}^{2}\right) \\
1 \leq|m| \equiv m_{3}+m_{4} \leq N_{z}, i=1,4, j=1,2
\end{gathered}
$$

where is denoted: $\lambda(t) \equiv\left(\lambda_{1}, \lambda_{2}, \lambda_{3}, \lambda_{4}\right),(m, \lambda(t)) \equiv m_{3} \lambda_{3}(t)+m_{4} \lambda_{4}(t),\left(e_{j}+m, \lambda(t)\right) \equiv \lambda_{j}(t)+m_{3} \lambda_{3}(t)+$ $+m_{4} \lambda_{4}(t)$; an asterisk $*$ above the sum sign indicates that the summation for $|m| \geq 1$ it occurs only over multi-indices $m=\left(0,0, m_{3}, m_{4}\right)$ with $m_{3} \neq m_{4}, e_{1}=(1,0,0,0), e_{2}=(0,1,0,0), \sigma=\left(\sigma_{1}, \sigma_{2}\right)$.

Note that here the degree $N_{z}$ of the polynomial $z(t, \tau, \sigma)$ relative to the exponentials $e^{\tau_{j}}$ depends on the element $z$. In addition, the elements of space $U$ depend on bounded in $\varepsilon>0$ terms of constants $\sigma_{1}=\sigma_{1}(\varepsilon)$ and $\sigma_{2}=\sigma_{2}(\varepsilon)$, and which do not affect the development of the algorithm described below, therefore, in the record of element (5) of this space $U$, we omit the dependence on $\sigma=\left(\sigma_{1}, \sigma_{2}\right)$ for brevity. We show that the class $M_{\varepsilon}=\left.U\right|_{\tau=\psi(t) / \varepsilon}$ is asymptotically invariant with respect to the operator $J$. The image of the operator on the element (5) of the space $U$ has the form 


$$
\begin{aligned}
J z(t, \tau)= & \int_{t_{0}}^{t} K(t, s) z_{0}(s) d s+\sum_{i=1}^{4} \int_{t_{0}}^{t} K(t, s) z_{i}(s) e^{\frac{1}{\varepsilon} \int_{t_{0}}^{s} \lambda_{i}(\theta) d \theta} d s+ \\
& +\sum_{2 \leq|m| \leq N_{z}}^{*} \int_{t_{0}}^{t} K(t, s) z^{m}(s) e^{\frac{1}{\varepsilon} \int_{t_{0}}^{s}(m, \lambda(\theta)) d \theta} d s+ \\
+ & \sum_{j=1}^{2} \sum_{1 \leq|m| \leq N_{z}}^{*} \int_{t_{0}}^{t} K(t, s) z^{e_{j}+m}(s) e^{\frac{1}{\varepsilon} \int_{t_{0}}^{s}\left(e_{j}+m, \lambda(\theta)\right) d \theta} d s .
\end{aligned}
$$

Integrating in parts, we will have

$$
\begin{gathered}
J_{i}(t, \varepsilon)=\int_{t_{0}}^{t} K(t, s) z_{i}(s) e^{\frac{1}{\varepsilon} \int_{t_{0}}^{s} \lambda_{i}(\theta) d \theta} d s=\varepsilon \int_{t_{0}}^{t} \frac{K(t, s) z_{i}(s)}{\lambda_{i}(s)} d e^{\frac{1}{\varepsilon} \int_{t_{0}}^{s} \lambda_{i}(\theta) d \theta}= \\
=\left.\varepsilon \frac{K(t, s) z_{i}(s)}{\lambda_{i}(s)} e^{\frac{1}{\varepsilon} \int_{t_{0}}^{s} \lambda_{i}(\theta) d \theta}\right|_{s=t_{0}} ^{s=t}-\varepsilon \int_{t_{0}}^{t}\left(\frac{\partial}{\partial s} \frac{K(t, s) z_{i}(s)}{\lambda_{i}(s)}\right) e^{\frac{1}{\varepsilon} \int_{t_{0}}^{s} \lambda_{i}(\theta) d \theta} d s= \\
=\varepsilon\left[\frac{K(t, t) z_{i}(t)}{\lambda_{i}(t)} e^{\frac{1}{\varepsilon} \int_{t_{0}}^{t} \lambda_{i}(\theta) d \theta}-\frac{K\left(t, t_{0}\right) z_{i}\left(t_{0}\right)}{\lambda_{i}\left(t_{0}\right)}\right]-\varepsilon \int_{t_{0}}^{t}\left(\frac{\partial}{\partial s} \frac{K(t, s) z_{i}(s)}{\lambda_{i}(s)}\right) e^{\frac{1}{\varepsilon} \int_{t_{0}}^{s} \lambda_{i}(\theta) d \theta} d s .
\end{gathered}
$$

Continuing this process further, we obtain the decomposition

$$
\begin{gathered}
J_{i}(t, \varepsilon)=\sum_{\nu=0}^{\infty}(-1)^{\nu} \varepsilon^{\nu+1}\left[\left(I_{i}^{\nu}\left(K(t, s) z_{i}(s)\right)\right)_{s=t} e^{\left.\frac{1}{\varepsilon} \int_{t_{0}}^{t} \lambda_{i}(\theta)\right) d \theta}-\left(I_{i}^{\nu}\left(K(t, s) z_{i}(s)\right)\right)_{s=t_{0}}\right], \\
I_{i}^{0}=\frac{1}{\lambda_{i}(s)} \cdot I_{i}^{\nu}=\frac{1}{\lambda_{i}(s)} I_{i}^{\nu-1} \quad(\nu \geq 1, i=\overline{1,4}) .
\end{gathered}
$$

Applying the integration operation in parts to integrals

$$
J_{m}(t, \varepsilon)=\int_{t_{0}}^{t} K(t, s)(s) e^{\frac{1}{\varepsilon} \int_{t_{0}}^{s}(m, \lambda(\theta)) d \theta} d s, J_{e_{j}+m}(t, \varepsilon)=\int_{t_{0}}^{t} K(t, s) z^{e_{j}+m}(s) e^{\frac{1}{\varepsilon} \int_{t_{0}}^{s}\left(e_{j}+m, \lambda(\theta)\right) d \theta} d s,
$$

we note that for all multi-indices $m=\left(0,0, m_{3}, m_{4}\right), m_{3} \neq m_{4}$, inequalities

$$
(m, \lambda(t)) \equiv m_{3} \lambda_{3}(t)+m_{4} \lambda_{4}(t) \neq 0 \forall t \in\left[t_{0}, T\right], m_{3}+m_{4} \geq 2
$$

are satisfied. In addition, for the same multi-indices we have

$$
\left(e_{j}+m, \lambda(t)\right) \neq 0 \forall t \in\left[t_{0}, T\right], j=1,2, m_{3} \neq m_{4},|m|=m_{3}+m_{4} \geq 1 .
$$

Indeed, if $\left(e_{1}+m, \lambda(t)\right)=0$ for some $t \in\left[t_{0}, T\right]$ and $m_{3} \neq m_{4}, m_{3}+m_{4} \geq 1$, then $m_{3} \lambda_{3}(t)+m_{4} \lambda_{4}(t)=$ $=-\lambda_{1}(t)=\lambda_{2}(t), m_{3}+m_{4} \geq 1$, which contradicts condition 2). And likewise, if $\left(e_{2}+m, \lambda(t)\right)=0$ with some $t \in\left[t_{0}, T\right]$ and $m_{3} \neq m_{4}, m_{3}+m_{4} \geq 1$, then $m_{3} \lambda_{3}(t)+m_{4} \lambda_{4}(t)=-\lambda_{1}(t)=\lambda_{2}(t), m_{3}+m_{4} \geq 1$, which also contradicts condition 2). Therefore, integration by parts in integrals $J_{m}(t, \varepsilon), J_{e_{j}+m}(t, \varepsilon)$ is possible. Performing it, we will have:

$$
\begin{gathered}
J_{m}(t, \varepsilon)=\int_{t_{0}}^{t} K(t, s) z^{m}(s) e^{\frac{1}{\varepsilon} \int_{t_{0}}^{s}(m, \lambda(\theta)) d \theta} d s=\varepsilon \int_{t_{0}}^{t} \frac{K(t, s) z^{m}(s)}{(m, \lambda(s))} d e^{\frac{1}{\varepsilon} \int_{t_{0}}^{s}(m, \lambda(\theta)) d \theta}= \\
=\varepsilon\left[\frac{K(t, t) z^{m}(t)}{(m, \lambda(t))} e^{\frac{1}{\varepsilon} \int_{t_{0}}^{t}(m, \lambda(\theta)) d \theta}-\frac{K\left(t, t_{0}\right) z^{m}\left(t_{0}\right)}{\left(m, \lambda\left(t_{0}\right)\right)}\right]-\varepsilon \int_{t_{0}}^{t} \frac{\partial}{\partial s} \frac{K(t, s) z^{m}(s)}{(m, \lambda(s))} e^{\frac{1}{\varepsilon} \int_{t_{0}}^{s}(m, \lambda(\theta)) d \theta} d s= \\
=\sum_{\nu=0}^{\infty}(-1)^{\nu} \varepsilon^{\nu+1}\left[\left(I_{m}^{\nu}\left(K(t, s) z^{m}(s)\right)\right)_{s=t} e^{\frac{1}{\varepsilon} \int_{t_{0}}^{t}(m, \lambda(\theta)) d \theta}-\left(I_{m}^{\nu}\left(K(t, s) z^{m}(s)\right)\right)_{s=t_{0}}\right] \\
I_{m}^{0}=\frac{1}{(m, \lambda(s))}, I_{m}^{\nu}=\frac{1}{(m, \lambda(s))} \frac{\partial}{\partial s} I_{m}^{\nu-1}(\nu \geq 1,|m| \geq 2) ; \\
J_{e_{j}+m}(t, \varepsilon)=\int_{t_{0}}^{t} K(t, s) z^{e_{j}+m}(s) e^{\frac{1}{\varepsilon} \int_{t_{0}}^{s}\left(e_{j}+m, \lambda(\theta)\right) d \theta} d s= \\
=\varepsilon \int_{t_{0}}^{t} \frac{K(t, s) z^{e_{j}+m}(s)}{\left(e_{j}+m, \lambda(s)\right)} d e^{\frac{1}{\varepsilon} \int_{t_{0}}^{s}\left(e_{j}+m, \lambda(\theta)\right) d \theta}=
\end{gathered}
$$




$$
\begin{gathered}
=-\varepsilon\left[\frac{K(t, t) z^{e_{j}+m}(t)}{\left(e_{j}+m, \lambda(t)\right)} e^{\frac{1}{\varepsilon} \int_{t_{0}}^{s}\left(e_{j}+m, \lambda(\theta)\right) d \theta}-\frac{K\left(t, t_{0}\right) z^{e_{j}+m}\left(t_{0}\right)}{\left(e_{j}+m, \lambda\left(t_{0}\right)\right)}\right]- \\
-\varepsilon \int_{t_{0}}^{t} \frac{\partial}{\partial s} \frac{K(t, s) z^{e_{j}+m}(s)}{\left(e_{j}+m, \lambda(s)\right)} e^{\frac{1}{\varepsilon} \int_{t_{0}}^{s}\left(e_{j}+m, \lambda(\theta)\right) d \theta} d s= \\
=\sum_{\nu=0}^{\infty}(-1)^{\nu} \varepsilon^{\nu+1}\left[\left(I_{j, m}^{\nu}\left(K(t, s) z^{e_{j}+m}(s)\right)\right)_{s=t} e^{\frac{1}{\varepsilon} \int_{t_{0}}^{t}\left(e_{j}+m, \lambda(\theta)\right) d \theta}-\right. \\
\left.-\left(I_{j, m}^{\nu}\left(K(t, s) z^{e_{j}+m}(s)\right)\right)_{s=t_{0}}\right], \\
I_{j, m}^{0}=\frac{1}{\left(e_{j}+m, \lambda(s)\right)}, \quad I_{j, m}^{\nu}=\frac{1}{\left(e_{j}+m, \lambda(s)\right)} \frac{\partial}{\partial s} I_{j, m}^{\nu-1}(\nu \geq 1,|m| \geq 1, j=1,2),
\end{gathered}
$$

Therefore, the image of the operator $J$ on the element (5) of the space $U$ is represented as a series

$$
\begin{gathered}
J z(t, \tau)=\int_{t_{0}}^{t} K(t, s) z_{0}(s) d s+\sum_{i=1}^{4} \sum_{\nu=0}^{\infty}(-1)^{\nu} \varepsilon^{\nu+1}\left[\left(I_{i}^{\nu}\left(K(t, s) z_{i}(s)\right)\right)_{s=t} e^{\left.\frac{1}{\varepsilon} \int_{t_{0}}^{t} \lambda_{i}(\theta)\right) d \theta}-\right. \\
\left.-\left(I_{i}^{\nu}\left(K(t, s) z_{i}(s)\right)\right)_{s=t_{0}}\right]+\sum_{\nu=0}^{\infty}(-1)^{\nu} \varepsilon^{\nu+1}\left[\left(I_{m}^{\nu}\left(K(t, s) z^{m}(s)\right)\right)_{s=t} e^{\frac{1}{\varepsilon} \int_{t_{0}}^{t}(m, \lambda(\theta)) d \theta}-\right. \\
\left.-\left(I_{m}^{\nu}\left(K(t, s) z^{m}(s)\right)\right)_{s=t_{0}}\right]+\sum_{j=1}^{2} \sum_{1 \leq|m| \leq N_{z}} \sum_{\nu=0}^{\infty}(-1)^{\nu} \varepsilon^{\nu+1}\left[\left(I_{j, m}^{\nu}\left(K(t, s) z^{e_{j}+m}(s)\right)\right)_{s=t} \times\right. \\
\left.\times e^{\frac{1}{\varepsilon} \int_{t_{0}}^{t}\left(e_{j}+m, \lambda(\theta)\right)}-\left(I_{j, m}^{\nu}\left(K(t, s) z^{e_{j}+m}(s)\right)\right)_{s=t_{0}}\right]_{\tau=\psi(t) / \varepsilon} .
\end{gathered}
$$

It is easy to show [22; 291-294] that this series converges asymptotically for $\varepsilon \rightarrow+0$ (uniformly in $t \in\left[t_{0}, T\right]$ ). This means that the class $M_{\varepsilon}$ is asymptotically invariant (for $\varepsilon \rightarrow+0$ ) with respect to the operator $J$.

We introduce operators $R_{\nu}: U \rightarrow U$, acting on each element $z(t, \tau) \in U$ of the form (5) according to the law:

$$
\begin{gathered}
R_{0} z(t, \tau)=\int_{t_{0}}^{t} K(t, s) z_{0}(s) d s \\
R_{1} z(t, \tau)=\sum_{i=1}^{4}\left[\left(I_{i}^{0}\left(K(t, s) z_{i}(s)\right)\right)_{s=t} e^{\tau_{i}}-\left(I_{i}^{0}\left(K(t, s) z_{i}(s)\right)\right)_{s=t_{0}}\right]+ \\
+\sum_{1 \leq|m| \leq N_{z}}^{*}\left(I_{m}^{0}\left(K(t, s) z^{m}(s)\right)\right)_{s=t} e^{(m, \tau)}-\left(I_{m}^{0}\left(K(t, s) z^{m}(s)\right)\right)_{s=t_{0}}+ \\
+\sum_{j=1}^{2} \sum_{1 \leq|m| \leq N_{z}}^{*}\left[\left(I_{j, m}^{0}\left(K(t, s) z^{e_{j}+m}(s)\right)\right)_{s=t} e^{\left(e_{j}+m, \tau\right)}-\left(I_{j, m}^{0}\left(K(t, s) z^{e_{j}+m}(s)\right)\right)_{s=t_{0}}\right] \\
R_{\nu+1} z(t, \tau)=\sum_{i=1}^{4}(-1)^{\nu} \varepsilon^{\nu+1}\left[\left(I_{i}^{\nu}\left(K(t, s) z_{i}(s)\right)\right)_{s=t} e^{\tau_{i}}-\left(I_{i}^{\nu}\left(K(t, s) z_{i}(s)\right)\right)_{s=t_{0}}\right]+ \\
+\sum_{\nu=0}^{*}(-1)^{\nu} \varepsilon^{\nu+1}\left[\left(I_{m}^{\nu}\left(K(t, s) z^{m}(s)\right)\right)_{s=t} e^{(m, \tau)}-\left(I_{m}^{\nu}\left(K(t, s) z^{m}(s)\right)\right)_{s=t_{0}}\right]+ \\
+\sum_{j=1}^{2} \sum_{1 \leq|m| \leq N_{z}}^{*}(-1)^{\nu}\left[\left(I_{j, m}^{\nu}\left(K(t, s) z^{e_{j}+m}(s)\right)\right)_{s=t} e^{\left(e_{j}+m, \tau\right)}-\left(I_{j, m}^{\nu}\left(K(t, s) z^{e_{j}+m}(s)\right)\right)_{s=t_{0}}\right], \nu \geq 1 .
\end{gathered}
$$

Now let $\tilde{z}(t, \tau, \varepsilon)$ be an arbitrary continuous function on $(t, \tau) \in\left[t_{0}, T\right] \times\left\{\tau: \operatorname{Re} \tau_{j} \leq 0, j=\overline{1,4}\right\}$ with asymptotic expansion

$$
\tilde{z}(t, \tau, \varepsilon)=\sum_{k=0}^{\infty} \varepsilon^{k} z_{k}(t, \tau), z_{k}(t, \tau) \in U
$$


converging as $\varepsilon \rightarrow+0$ (uniformly in $(t, \tau) \in\left[t_{0}, T\right] \times\left\{\tau: R e \tau_{j} \leq 0, j=\overline{1,4}\right\}$ ). Then the image $J \tilde{z}(t, \tau, \varepsilon)$ of this function is decomposed into an asymptotic series

$$
J \tilde{z}(t, \tau, \varepsilon)=\sum_{k=0}^{\infty} \varepsilon^{k} J z_{k}(t, \tau)=\left.\sum_{r=0}^{\infty} \varepsilon^{r} \sum_{s=0}^{r} R_{r-s} z_{s}(t, \tau)\right|_{\tau=\psi(t) / \varepsilon} .
$$

This equality is the basis for introducing an extension of an operator $J$ on series of the form (7):

$$
\tilde{J} \tilde{z}(t, \tau, \varepsilon) \equiv \tilde{J}\left(\sum_{k=0}^{\infty} \varepsilon^{k} z_{k}(t, \tau)\right) \triangleq \sum_{r=0}^{\infty} \varepsilon^{r} \sum_{s=0}^{r} R_{r-s} z_{s}(t, \tau) .
$$

Although the operator (8) is formally defined, its utility is obvious, since in practice it is usual to construct the $N$-th approximation of the asymptotic solution of the problem (2), in which impose only $N$-th partial sums of the series (7), which have not a formal, but a true meaning. Now you can write a problem that is completely regularized with respect to the original problem (2):

$$
\begin{gathered}
\varepsilon \frac{\partial \tilde{z}}{\partial t}+\sum_{j=1}^{4} \lambda_{j}(t) \frac{\partial \tilde{z}}{\partial \tau_{j}}-A(t) \tilde{z}-\varepsilon \frac{g(t)}{2}\left(e^{\tau_{3}} \sigma_{1}+e^{\tau_{4}} \sigma_{2}\right) B \tilde{z}-\tilde{J} \tilde{z}=h(t), \\
\left.\tilde{z}(t, \tau, \varepsilon)\right|_{t=t_{0}, \tau=0}=z^{0}, t \in\left[t_{0}, T\right] .
\end{gathered}
$$

\section{Iterative problems and their solvability in space solution of the first iterative problem}

Substituting the series (7) into (9) and equating the coefficients with the same degrees, we obtain the following iterative problems:

$$
\begin{gathered}
L z_{0}(t, \tau) \equiv \sum_{j=1}^{4} \lambda_{j}(t) \frac{\partial z_{0}}{\partial \tau_{j}}-A(t) z_{0}-R_{0} z_{0}=h(t), z_{0}\left(t_{0}, 0\right)=z^{0} \\
L z_{1}(t, \tau)=-\frac{\partial z_{0}}{\partial t}+\frac{g(t)}{2}\left(e^{\tau_{3}} \sigma_{1}+e^{\tau_{4}} \sigma_{2}\right) B(t) z_{0}+R_{1} z_{0}, z_{1}\left(t_{0}, 0\right)=0 ; \\
L z_{2}(t, \tau)=-\frac{\partial z_{1}}{\partial t}+\frac{g(t)}{2}\left(e^{\tau_{3}} \sigma_{1}+e^{\tau_{4}} \sigma_{2}\right) B(t) z_{1}+R_{1} z_{1}+R_{2} z_{0}, z_{0}\left(t_{0}, 0\right)=0 ; \\
\ldots \\
L z_{k}(t, \tau)=-\frac{\partial z_{k-1}}{\partial t}+\frac{g(t)}{2}\left(e^{\tau_{3}} \sigma_{1}+e^{\tau_{4}} \sigma_{2}\right) B(t) z_{k-1}+R_{k} z_{0}+\ldots+R_{1} z_{k-1}, \quad z_{k}\left(t_{0}, 0\right)=0, k \geq 1 .
\end{gathered}
$$

Each of the iterative problems $\left(10_{k}\right)$ can be written as

$$
L z(t, \tau) \equiv \sum_{j=1}^{4} \lambda_{j}(t) \frac{\partial z}{\partial \tau_{j}}-A(t) z-R_{0} z=H(t, \tau), z\left(t_{0}, 0\right)=z^{*}
$$

where

$$
H(t, \tau)=H_{0}(t)+\sum_{i=1}^{4} H_{i}(t) e^{\tau_{i}}+\sum_{2 \leq|m| \leq N_{z}}^{*} H^{m}(t) e^{(m, \tau)}+\sum_{j=1}^{2} \sum_{1 \leq|m| \leq N_{H}}^{*} H^{e_{j}+m}(t) e^{\left(e_{j}+m, \tau\right)}
$$

is the known vector function of space $U, z^{*}$ is the known constant vector of the complex space $\mathcal{C}^{2}$, and the operator $R_{0}$ has the form (see $\left.\left(6_{0}\right)\right)$

$$
\begin{aligned}
& R_{0} z \equiv R_{0}\left(z_{0}(t)+\sum_{i=1}^{4} z_{i}(t) e^{\tau_{i}}+\sum_{2 \leq|m| \leq N_{z}}^{*} z^{m}(t) e^{(m, \tau)}+\right. \\
& \left.+\sum_{j=1}^{2} \sum_{0 \leq|m| \leq N_{z}}^{*} z^{e_{j}+m}(t) e^{\left(e_{j}+m, \tau\right)}\right) \triangleq \int_{t_{0}}^{t} K(t, s) z_{0}(s) d s .
\end{aligned}
$$


In the future, we will need $\lambda_{j}(t)$-eigenvectors of the matrix $A(t)$ :

$$
\varphi_{1}(t)=\left(\begin{array}{c}
1 \\
-i \omega(t)
\end{array}\right), \varphi_{2}(t)=\left(\begin{array}{c}
1 \\
+i \omega(t)
\end{array}\right),
$$

and also $\bar{\lambda}_{j}(t)$-eigenvectors of the matrix $A^{*}(t)$ :

$$
\chi_{1}(t)=\left(\begin{array}{c}
1 \\
-\frac{i}{\omega(t)}
\end{array}\right), \chi_{2}(t)=\left(\begin{array}{c}
1_{i} \\
+\frac{i}{\omega(t)}
\end{array}\right) .
$$

These vectors form a biorthogonal system, i.e.

$$
\left(\varphi_{k}(t), \chi_{j}(t)\right)=\left\{\begin{array}{l}
2, k=j, \\
0, k \neq j
\end{array} \quad(k, j=1,2) .\right.
$$

We introduce scalar (for each $t \in\left[t_{0}, T\right]$ ) product in space $U$ :

$$
\begin{aligned}
& <z, w>\equiv \\
& \equiv<z_{0}(t)+\sum_{i=1}^{4} z_{i}(t) e^{\tau_{i}}+\sum_{2 \leq|m| \leq N_{z}}^{*} z^{m}(t) e^{(m, \tau)}+\sum_{j=1}^{2} \sum_{1 \leq|m| \leq N_{z}}^{*} z^{e_{j}+m}(t) e^{\left(e_{j}+m, \tau\right)}, \\
& w_{0}(t)+\sum_{i=1}^{4} w_{i}(t) e^{\tau_{i}}+\sum_{2 \leq|m| \leq N_{w}}^{*} w^{m}(t) e^{(m, \tau)}+\sum_{j=1}^{2} \sum_{1 \leq|m| \leq N_{w}}^{*} w^{e_{j}+m}(t) e^{\left(e_{j}+m, \tau\right)}>\triangleq \\
& \triangleq\left(z_{0}(t), w_{0}(t)\right)+\sum_{i=1}^{4}\left(z_{i}(t), w_{i}(t)\right)+ \\
& +\sum_{2 \leq|m| \leq \min \left(N_{z}, N_{w}\right)}^{*}\left(z^{m}(t), w^{m}(t)\right)+\sum_{j=1}^{2} \sum_{1 \leq|m| \leq \min \left(N_{z}, N_{w}\right)}^{*}\left(z^{e_{j}+m}(t), w^{e_{j}+m}(t)\right),
\end{aligned}
$$

where we denote by $(*, *)$ the usual scalar product in the complex space $\mathbb{C}^{2}$. Let us prove the following statement.

Theorem 1. Let conditions 1) and 2) be fulfilled and the right-hand side $H(t, \tau)=H_{0}(t)+\sum_{i=1}^{4} H_{i}(t) e^{\tau_{i}}+$ $+\sum_{2 \leq|m| \leq N_{z}}^{*} H^{m}(t) e^{(m, \tau)}+\sum_{j=1}^{2} \sum_{1 \leq|m| \leq N_{H}}^{*} H^{e_{j}+m}(t) e^{\left(e_{j}+m, \tau\right)}$ of system (10) belongs to the space $U$. Then the system (10) is solvable in $U$, if and only if

$$
<H(t, \tau), \chi_{k}(t) e^{\tau_{k}}>\equiv 0, k=1,2, \forall t \in\left[t_{0}, T\right] .
$$

Proof. We will determine the solution of system (10) as an element (5) of the space $U$ :

$$
\begin{gathered}
z(t, \tau)=z_{0}(t)+\sum_{i=1}^{4} z_{i}(t) e^{\tau_{i}}+\sum_{2 \leq|m| \leq N_{z}}^{*} z^{m}(t) e^{(m, \tau)}+ \\
+\sum_{j=1}^{2} \sum_{1 \leq|m| \leq N_{H}}^{*} z^{e_{j}+m}(t) e^{\left(e_{j}+m, \tau\right)} \equiv \\
\equiv z_{0}(t)+\sum_{i=1}^{4} z_{i}(t) e^{\tau_{i}}+\sum_{2 \leq|m| \leq N_{z}}^{*} z^{m}(t) e^{(m, \tau)}+\sum_{k=1}^{2} \sum_{2 \leq\left|m^{k}\right| \leq N_{H}}^{*} z^{m^{k}}(t) e^{\left(m^{k}, \tau\right)},
\end{gathered}
$$

where for convenience are introduced multi-indices

$$
m^{1}=e_{1}+m \equiv\left(1,0, m_{3}, m_{4}\right), m^{2}=e_{2}+m \equiv\left(0,1, m_{3} !, m_{4}\right),\left|m^{k}\right|=1+m_{3}+m_{4} \geq 2,
$$

$m_{3}$ and $m_{4}$ are non-negative integer numbers. Substituting (12) into system (10), we will have

$$
\begin{aligned}
& \sum_{i=1}^{4}\left[\lambda_{i}(t) I-A(t)\right] z_{i}(t) e^{\tau_{i}}+\sum_{2 \leq|m| \leq N_{z}}^{*}[(m, \lambda(t)) I-A(t)] z^{m}(t) e^{(m, \tau)}+ \\
& +\sum_{k=1}^{2} \sum_{2 \leq\left|m^{k}\right| \leq N_{H}}^{*}\left[\left(m^{k}, \lambda(t)\right) I-A(t)\right] z^{m^{k}}(t) e^{\left(m^{k}, \tau\right)}-A(t) z_{0}(t)-\int_{t_{0}}^{t} K(t, s) z_{0}(s) d s= \\
& =H_{0}(t)+\sum_{i=1}^{4} H_{i}(t) e^{\tau_{i}}+\sum_{2 \leq|m| \leq N_{z}}^{*} H^{m}(t) e^{(m, \tau)}+\sum_{k=1}^{2} \sum_{2 \leq\left|m^{k}\right| \leq N_{H}}^{*} H^{m^{k}}(t) e^{\left(m^{k}, \tau\right)} .
\end{aligned}
$$


Equating here the free terms and coefficients separately for identical exponents, we obtain the following systems of equations:

$$
\begin{gathered}
-A(t) z_{0}(t)-\int_{t_{0}}^{t} K(t, s) z_{0}(s) d s=H_{0}(t), \\
{\left[\lambda_{i}(t) I-A(t)\right] z_{i}(t)=H_{i}(t), i=\overline{1,4} ;} \\
{[(m, \lambda(t)) I-A(t)] z^{m}(t)=H^{m}(t), m_{3} \neq m_{4}, 2 \leq|m| \leq N_{H} ;} \\
{\left[\left(m^{k}, \lambda(t)\right) I-A(t)\right] z^{m^{k}}(t)=H^{m^{k}}(t), m_{3} \neq m_{4}, 2 \leq\left|m^{k}\right| \leq N_{H}, k=1,2 .}
\end{gathered}
$$

Since the matrix $A(t)$ is reversible, the system (13) can be written as

$$
z_{0}(t)=\int_{t_{0}}^{t}\left(-A^{-1}(t) K(t, s)\right) z_{0}(s) d s-A^{-1}(t) H_{0}(t) .
$$

Due to the smoothness of the kernel $-A^{-1}(t) K(t, s)$ and heterogeneity $-A^{-1}(t) H_{0}(t)$, this Volterra integral system has a unique solution $z_{0}(t) \in C^{\infty}\left(\left[t_{0}, T\right], \mathbb{C}^{2}\right)$. The systems $\left(13_{3}\right)$ and $\left(13_{4}\right)$ also have unique solutions

$$
z_{i}(t)=\left[\lambda_{i}(t) I-A(t)\right]^{-1} H_{i}(t) \in C^{\infty}\left(\left[t_{0}, T\right], \mathbb{C}^{2}\right), i=3,4,
$$

since $\lambda_{3}(t), \lambda_{4}(t)$ do not belong to the spectrum of the matrix $A(t)$. Systems $\left(13_{1}\right)$ and $\left(13_{2}\right)$ are solvable in space $C^{\infty}\left(\left[t_{0}, T\right], \mathbb{C}^{2}\right)$ if and only if there are identities

$$
\left(H_{i}(t), \chi_{i}(t)\right) \equiv 0 \forall t \in\left[t_{0}, T\right], i=1,2 .
$$

It is not difficult to see that these identities coincide with identities $(11)$. Further, since $(m, \lambda(t)) \equiv m_{3} \lambda_{3}(t)+$ $+m_{4} \lambda_{4}(t) \neq \lambda_{j}(t), j=1,2,|m|=m_{3}+m_{4} \geq 2, m_{3} \neq m_{4}$ (see condition 2) the absence of resonance), the system $\left(13_{m}\right)$ has a unique solution

$$
z^{m}(t)=[(m, \lambda(t)) I-A(t)]^{-1} H^{m}(t), 2 \leq|m| \leq N_{H} \in C^{\infty}\left(\left[t_{0}, T\right], \mathbb{C}^{2}\right) .
$$

We now consider systems (14). Let us show that when $\left|m^{k}\right| \geq 2$ the functions $\left(m^{k}, \lambda(t)\right)$ are not eigenvalues of the matrix $A(t)$. Indeed, let $\left(m^{1}, \lambda(t)\right)=\lambda_{1}(t),\left|m^{1}\right| \geq 2$. Then

$$
\lambda_{1}(t)+m_{3} \lambda_{3}(t)+m_{4} \lambda_{4}(t)=\lambda_{2}(t), m_{3}+m_{4} \geq 1,
$$

which contradicts condition 2$)$ the absence of resonance. And likewise, equality $\left(m^{2}, \lambda(t)\right)=\lambda_{1}(t),\left|m^{2}\right| \geq 2$ $m_{3}+m_{4} \geq 1$ cannot be fulfilled.

Therefore, when $\left|m^{k}\right| \geq 2$ the matrix $\left(m^{k}, \lambda(t)\right) I-A(t)$ is reversible, we get a unique solution of system (14) for $\left|m^{k}\right| \geq 2$ in the class $C^{\infty}\left(\left[t_{0}, T\right], \mathcal{C}^{2}\right)$ :

$$
z^{m^{k}}(t)=\left[\left(m^{k}, \lambda(t)\right) I-A(t)\right]^{-1} H^{m^{k}}(t), 2 \leq\left|m^{k}\right| \leq N_{H}, k=1,2 .
$$

Thus, condition (11) is necessary and sufficient for the solvability of system (10) in the space $U$. The theorem is proved.

Remark 1. If identity (11) holds, then under conditions 1) and 2), system (10) has the following solution in the space $U$ :

$$
\begin{gathered}
z(t, \tau)=z_{0}(t)+\sum_{k=1}^{2} \alpha_{k}(t) \varphi_{k}(t) e^{\tau_{k}}+\frac{\left(H_{1}(t), \chi_{2}(t)\right)}{\lambda_{1}(t)-\lambda_{2}(t)} \varphi_{2}(t) e^{\tau_{1}}+ \\
+\frac{\left(H_{2}(t), \chi_{1}(t)\right)}{\lambda_{2}(t)-\lambda_{1}(t)} \varphi_{1}(t) e^{\tau_{2}}+\sum_{i=3}^{4}\left[\lambda_{i}(t) I-A(t)\right]^{-1} H_{i}(t) e^{\tau_{i}}+ \\
\quad+\sum_{2 \leq|m| \leq N_{H}}^{*}[(m, \lambda(t)) I-A(t)]^{-1} H^{m}(t) e^{(m, \tau)}+ \\
+\sum_{k=1}^{2} \sum_{1 \leq|m| \leq N_{H}}^{*}\left[\left(e_{k}+m, \lambda(t)\right) I-A(t)\right]^{-1} H^{e_{k}+m}(t) e^{\left(e_{k}+m, \tau\right)}
\end{gathered}
$$

where $\alpha_{k}(t) \in C^{\infty}\left(\left[t_{0}, T\right], \mathbb{C}^{1}\right)$ are arbitrary functions, $k=1,2, z_{0}(t)$ is the solution of an integral $\operatorname{system}\left(13_{0}\right)$, $m \equiv\left(0,0, m_{3}, m_{4}\right), m_{3} \neq m_{4},|m|=m_{3}+m_{4} \geq 1$. 
3. The unique solvability of the general iterative problem in the space U. Residual term theorem

Let us proceed to the description of the conditions for the unique solvability of system (10) in space Along with problem (10), we consider the system

$$
L w(t, \tau)=-\frac{\partial z}{\partial t}+\frac{g(t)}{2}\left(e^{\tau_{3}} \sigma_{1}+e^{\tau_{4}} \sigma_{2}\right) B(t) z+Q(t, \tau)
$$

where $z=z(t, \tau)$ is the solution (15) of the system (10), $Q(t, \tau)$ is the well-known function of the space $U$. The right part of this system:

$$
\begin{gathered}
G(t, \tau) \equiv-\frac{\partial z}{\partial t}+\frac{g(t)}{2}\left(e^{\tau_{3}} \sigma_{1}+e^{\tau_{4}} \sigma_{2}\right) B(t) z+Q(t, \tau)=-\frac{\partial}{\partial t}\left[z_{0}(t)+\sum_{i=1}^{4} z_{i}(t) e^{\tau_{i}}+\right. \\
\left.+\sum_{2 \leq|m| \leq N_{z}}^{*} z^{m}(t) e^{(m, \tau)}+\sum_{j=1}^{2} \sum_{1 \leq|m| \leq N_{z}}^{*} z^{e_{j}+m}(t) e^{\left(e_{j}+m, \tau\right)}\right]+\frac{g(t)}{2}\left(e^{\tau_{3}} \sigma_{1}+e^{\tau_{4}} \sigma_{2}\right) B(t) \times \\
\times\left[z_{0}(t)+\sum_{i=1}^{4} z_{i}(t) e^{\tau_{i}}+\sum_{2 \leq|m| \leq N_{z}}^{*} z^{m}(t) e^{(m, \tau)}+\sum_{j=1}^{2} \sum_{1 \leq|m| \leq N_{z}}^{*} z^{e_{j}+m}(t) e^{\left(e_{j}+m, \tau\right)}\right]+Q(t, \tau),
\end{gathered}
$$

may not belong to space $U$, if $z=z(t, \tau) \in U$. Indeed, taking into account the form (15) of the function $z=z(t, \tau) \in U$, we will have

$$
\begin{gathered}
Z(t, \tau) \equiv G(t, \tau)+\frac{\partial z}{\partial t}=\frac{g(t)}{2}\left(e^{\tau_{3}} \sigma_{1}+e^{\tau_{4}} \sigma_{2}\right) B(t)\left[z_{0}(t)+\right. \\
\left.+\sum_{i=1}^{4} z_{i}(t) e^{\tau_{i}}+\sum_{2 \leq|m| \leq N_{z}}^{*} z^{m}(t) e^{(m, \tau)}+\sum_{j=1}^{2} \sum_{1 \leq|m| \leq N_{z}}^{*} z^{e_{j}+m}(t) e^{\left(e_{j}+m, \tau\right)}\right]= \\
=\frac{g(t)}{2} B(t) z_{0}(t)\left(e^{\tau_{3}} \sigma_{1}+e^{\tau_{4}} \sigma_{2}\right)+\sum_{i=3}^{4} \frac{g(t)}{2} B(t) z_{i}(t)\left(e^{\tau_{i}+\tau_{3}} \sigma_{1}+e^{\tau_{i}+\tau_{4}} \sigma_{2}\right)+ \\
+\sum_{k=1}^{2} \frac{g(t)}{2} B(t) z_{k}(t)\left(e^{\tau_{k}+\tau_{3}} \sigma_{1}+e^{\tau_{k}+\tau_{4}} \sigma_{2}\right)+ \\
+\frac{g(t)}{2}\left(e^{\tau_{3}} \sigma_{1}+e^{\tau_{4}} \sigma_{2}\right) B(t)\left[\sum_{2 \leq|m| \leq N_{z}}^{*} z^{m}(t) e^{(m, \tau)}+\sum_{j=1}^{2} \sum_{1 \leq|m| \leq N_{z}}^{*} z^{e_{j}+m}(t) e^{\left(e_{j}+m, \tau\right)}\right]+
\end{gathered}
$$

Here are terms with exponents

$$
\begin{aligned}
& e^{\tau_{4}+\tau_{3}}=\left.e^{(m, \tau)}\right|_{m=(0,0,1,1)}, \\
& e^{\tau_{3}+(m, \tau)}\left(\text { if } m_{3}+1=m_{4}\right), e^{\tau_{4}+(m, \tau)}\left(\text { if } m_{4}+1=m_{3}\right), \\
& e^{\tau_{3}+\left(e_{1}+m, \tau\right)}\left(\text { if } m_{3}+1=m_{4}\right), e^{\tau_{4}+\left(e_{2}+m, \tau\right)}\left(\text { if } m_{4}+1=m_{3}\right)
\end{aligned}
$$

do not belong to space $U$, since in multi-index $m=\left(0,0, m_{3}, m_{4}\right)$ of the space $U$ must be $m_{3} \neq m_{4}, m_{3}+m_{4} \geq 1$. Then, according to the well-known theory [3;234], we embed these terms in the space $U$ according to the following rule $(\operatorname{see}(*))$ :

$$
\begin{aligned}
& \widehat{e^{\tau_{4}+\tau_{3}}}=e^{0}=1, \widehat{e^{\widehat{\tau_{3}+(m, \tau)}}}= \\
& =e^{0}=1\left(m_{3}+1=m_{4}, m_{3} \neq m_{4}\right), \widehat{e^{\tau+(m, \tau)}}=e^{0}=1\left(m_{4}+1=m_{3}, m_{3} \neq m_{4}\right), \\
& \left.\left.e^{\tau_{3}+\left(e_{1}+m\right.}, \tau\right)=e^{\tau_{1}}\left(m_{3}+1=m_{4}, m_{3} \neq m_{4}\right), e^{\tau_{4}} \widehat{+\left(e_{2}+m\right.}, \tau\right)=e^{\tau_{2}}\left(m_{4}+1=m_{3}, m_{3} \neq m_{4}\right) .
\end{aligned}
$$

In $Z(t, \tau)$ need of embedding only the terms

$$
\begin{aligned}
& M(t, \tau) \equiv \sum_{i=3}^{4} \frac{g(t)}{2} B(t) z_{i}(t)\left(e^{\tau_{i}+\tau_{3}} \sigma_{1}+e^{\tau_{i}+\tau_{4}} \sigma_{2}\right)+\sum_{k=1}^{2} \frac{g(t)}{2} B(t) z_{k}(t)\left(e^{\tau_{k}+\tau_{3}} \sigma_{1}+e^{\tau_{k}+\tau_{4}} \sigma_{2}\right), \\
& S(t, \tau) \equiv \frac{g(t)}{2}\left(e^{\tau_{3}} \sigma_{1}+e^{\tau_{4}} \sigma_{2}\right) B(t)\left[\sum_{2 \leq|m| \leq N_{z}}^{*} z^{m}(t) e^{(m, \tau)}+\sum_{j=1}^{2} \sum_{1 \leq|m| \leq N_{z}}^{*} z^{e_{j}+m}(t) e^{\left(e_{j}+m, \tau\right)}\right] .
\end{aligned}
$$


We describe this embedding in more detail, taking into account formulas $(* *)$ :

$$
\begin{gathered}
M(t, \tau) \equiv \sum_{k=1}^{2} \frac{g(t)}{2} B(t) z_{k}(t)\left(e^{\tau_{k}+\tau_{3}} \sigma_{1}+e^{\tau_{k}+\tau_{4}} \sigma_{2}\right)+ \\
+\sum_{i=3}^{4} \frac{g(t)}{2} B(t) z_{i}(t)\left(e^{\tau_{i}+\tau_{3}} \sigma_{1}+e^{\tau_{i}+\tau_{4}} \sigma_{2}\right)= \\
=\frac{g(t)}{2} B(t)\left[z_{1}(t) e^{\tau_{1}+\tau_{3}} \sigma_{1}+z_{1}(t) e^{\tau_{1}+\tau_{4}} \sigma_{2}+z_{2}(t) e^{\tau_{2}+\tau_{3}} \sigma_{1}+z_{2}(t) e^{\tau_{2}+\tau_{4}} \sigma_{2}+\right. \\
\left.+z_{3}(t) e^{2 \tau_{3}} \sigma_{1}+z_{3}(t) e^{\tau_{3}+\tau_{4}} \sigma_{2}+z_{4}(t) e^{\tau_{4}+\tau_{3}} \sigma_{1}+z_{4}(t) e^{2 \tau_{4}} \sigma_{2}\right] \Rightarrow \\
\Rightarrow \quad \widehat{M}(t, \tau)=\frac{g(t)}{2} B(t)\left[z_{1}(t) e^{\tau_{1}+\tau_{3}} \sigma_{1}+z_{1}(t) e^{\tau_{1}+\tau_{4}} \sigma_{2}+z_{2}(t) e^{\tau_{2}+\tau_{3}} \sigma_{1}+\right. \\
+z_{2}(t) e^{\tau_{2}+\tau_{4}} \sigma_{2}+z_{3}(t) e^{2 \tau_{3}} \sigma_{1}+z_{3}(t) \sigma_{2}+z_{4}(t) \sigma_{1}+z_{4}(t) e^{2 \tau_{4}} \sigma_{2}
\end{gathered}
$$

(note that in $\widehat{M}(t, \tau)$ there are no members containing $e^{\tau_{1}}, e^{\tau_{2}}$ measurement exponents $|m|=1$ );

$$
\begin{aligned}
& S(t, \tau) \equiv \frac{g(t)}{2}\left(e^{\tau_{3}} \sigma_{1}+e^{\tau_{4}} \sigma_{2}\right) B(t)\left[\sum_{2 \leq|m| \leq N_{z}}^{*} z^{m}(t) e^{(m, \tau)}+\right. \\
& \left.+\sum_{j=1}^{2} \sum_{1 \leq|m| \leq N_{z}}^{*} z^{e_{j}+m}(t) e^{\left(e_{j}+m, \tau\right)}\right]= \\
& =\frac{g(t)}{2} B(t)\left[\sum_{2 \leq|m| \leq N_{z}}^{*} z^{m}(t)\left(e^{\tau_{3}+(m, \tau)} \sigma_{1}+e^{\tau_{4}+(m, \tau)} \sigma_{2}\right)+\right. \\
& +\sum_{1 \leq|m| \leq N_{z}}^{*} z^{e_{1}+m}(t)\left(e^{\left(e_{1}+m, \tau\right)+\tau_{3}} \sigma_{1}+e^{\left(e_{1}+m, \tau\right)+\tau_{4}} \sigma_{2}\right)+ \\
& \left.+\sum_{1 \leq|m| \leq N_{z}}^{*} z^{e_{2}+m}(t)\left(e^{\left(e_{2}+m, \tau\right)+\tau_{3}} \sigma_{1}+e^{\left(e_{2}+m, \tau\right)+\tau_{4}} \sigma_{2}\right)\right] \Rightarrow \widehat{S}(t, \tau)= \\
& =\frac{g(t)}{2} B(t) \sum_{\substack{2 \leq|m| \leq N_{z}, m_{3}+1=m_{4}}} z^{m}(t) \sigma_{1}+\sum_{\substack{2 \leq|m| \leq N_{z}, m_{4}+1=m_{3}}} z^{m}(t) \sigma_{2}+\sum_{\substack{2 \leq|m| \leq N_{z}, m_{3}+1 \neq m_{4}, m_{4}+1 \neq m_{3}}}^{*} z^{m}(t) e^{(m, \tau)}+ \\
& +\left(\sum_{\begin{array}{c}
1 \leq|m| \leq N_{z}, \\
m_{3}+1=m_{4}
\end{array}} z^{e_{1}+m}(t) \sigma_{1}+\sum_{\substack{1 \leq|m| \leq N_{z}, m_{4}+1=m_{3}}} z^{e_{1}+m}(t) \sigma_{2}\right) e^{\tau_{1}}+ \\
& +\left(\sum_{\substack{1 \leq|m| \leq N_{z}, m_{3}+1=m_{4}}} z^{e_{2}+m}(t) \sigma_{1}+\sum_{\substack{1 \leq|m| \leq N_{z}, m_{4}+1=m_{3}}} z^{e_{2}+m}(t) \sigma_{2}\right) e^{\tau_{2}}+ \\
& \left.+\sum_{j=1}^{2} \sum_{1 \leq|m| \leq N_{z},}^{*} z^{e_{j}+m}(t) e^{\left(e_{j}+m, \tau\right)}\right] . \\
& m_{3}+1 \neq m_{4}, m_{4}+1 \neq m_{3}
\end{aligned}
$$


After embedding, the right-hand side of system (20) will look like

$$
\begin{gathered}
\widehat{G}(t, \tau)=-\frac{\partial}{\partial t}\left[z_{0}(t)+\sum_{i=1}^{4} z_{i}(t) e^{\tau_{i}}+\sum_{2 \leq|m| \leq N_{z}}^{*} z^{m}(t) e^{(m, \tau)}+\right. \\
\left.+\sum_{j=1}^{2} \sum_{1 \leq|m| \leq N_{z}}^{*} z^{e_{j}+m}(t) e^{\left(e_{j}+m, \tau\right)}\right]+\widehat{M}(t, \tau)+\widehat{S}(t, \tau)+Q(t, \tau),
\end{gathered}
$$

moreover, in $\widehat{S}(t, \tau)$ the coefficients at $e^{\tau_{1}}, e^{\tau_{2}}$ do not depend on $z_{k}(t), k=1,2$. As indicated in [3], the embedding $G(t, \tau) \rightarrow \widehat{G}(t, \tau)$ will not affect the accuracy of the construction of asymptotic solutions of problem (2), since $\left.\left.\widehat{Z}(t, \tau)\right|_{\tau=\psi(t) / \varepsilon} \equiv Z(t, \tau)\right|_{\tau=\psi(t) / \varepsilon}$.

Theorem 2. Let conditions 1) and 2) be fulfilled and the right-hand side $H(t, \tau)=H_{0}(t)+\sum_{i=1}^{4} H_{i}(t) e^{\tau_{i}}+$ $+\sum_{2 \leq|m| \leq N_{z}}^{*} H^{m}(t) e^{(m, \tau)}+\sum_{j=1}^{2} \sum_{1 \leq|m| \leq N_{H}}^{*} H^{e_{j}+m}(t) e^{\left(e_{j}+m, \tau\right)} \in U$ of system (10) satisfy condition (11). Then problem (10) under additional conditions

$$
<\widehat{G}(t, \tau), \chi_{k}(t) e^{\tau_{k}}>\equiv 0 \forall t \in\left[t_{0}, T\right], k=1,2,
$$

where

$$
Q(t, \tau)=Q_{0}(t)+\sum_{k=1}^{4} Q_{k}(t) e^{\tau_{k}}+\sum_{2 \leq|m| \leq N_{z}}^{*} Q^{m}(t) e^{(m, \tau)}+\sum_{k=1}^{2} \sum_{1 \leq|m| \leq N_{Q}}^{*} Q^{e_{k}+m}(t) e^{\left(e_{k}+m, \tau\right)}
$$

is the known vector function of space $U$, is uniquely solvable in $U$.

Proof. Since the right-hand side of system (10) satisfies condition (11), this system has a solution in space $U$ in the form (15), where $\alpha_{k}(t) \in C^{\infty}\left(\left[t_{0}, T\right], \mathbb{C}^{1}\right)$ are arbitrary functions so far, $k=1,2$. Submit (15) to the initial condition $z\left(t_{0}, 0\right)=z^{*}$. We get $\sum_{k=1}^{2} \alpha_{k}\left(t_{0}\right) \varphi_{k}\left(t_{0}\right)=z_{*}$, where denoted

$$
\begin{gathered}
z_{*}=z^{*}+A^{-1}\left(t_{0}\right) H_{0}\left(t_{0}\right)-\sum_{i=3}^{4}\left[\lambda_{i}\left(t_{0}\right) I-A\left(t_{0}\right)\right]^{-1} H_{i}\left(t_{0}\right)- \\
-\frac{\left(H^{e_{1}}\left(t_{0}\right), \chi_{2}\left(t_{0}\right)\right)}{\lambda_{1}\left(t_{0}\right)-\lambda_{2}\left(t_{0}\right)} \varphi_{2}\left(t_{0}\right)-\frac{\left(H^{e_{2}}\left(t_{0}\right), \chi_{1}\left(t_{0}\right)\right)}{\lambda_{2}\left(t_{0}\right)-\lambda_{1}\left(t_{0}\right)} \varphi_{1}\left(t_{0}\right)- \\
-\sum_{2 \leq|m| \leq N_{z}}^{*} z^{m}\left(t_{0}\right)-\sum_{k=1}^{2} \sum_{1 \leq\left|m^{k}\right| \leq N_{H}}^{*}\left[\left(m^{k}, \lambda\left(t_{0}\right)\right) I-A\left(t_{0}\right)\right]^{-1} H^{m^{k}}\left(t_{0}\right) .
\end{gathered}
$$

Multiplying the equality $\sum_{k=1}^{2} \alpha_{k}\left(t_{0}\right) \varphi_{k}\left(t_{0}\right)=z_{*}$ scalarly by $\chi_{j}\left(t_{0}\right)$ and taking into account the biorthogonality of the systems $\left\{\varphi_{k}(t)\right\}$ and $\left\{\chi_{j}(t)\right\}$, we find the values $\alpha_{k}\left(t_{0}\right)=\frac{1}{2}\left(z_{*}, \chi_{k}\left(t_{0}\right)\right), k=1,2$. Now we submit the solution (15) to the condition of orthogonality (17). Considering that under these conditions, scalar multiplication performed by vector functions $\chi_{k}(t) e^{\tau_{k}}$, containing only exponents $e^{\tau_{k}}, k=1,2$, it is necessary to keep in the expression $\widehat{G}(t, \tau)$ only terms with exponents $e^{\tau_{1}}$ and $e^{\tau_{2}}$. Then condition (17) takes the form

$$
\begin{aligned}
& <-\frac{\partial}{\partial t}\left(\sum_{k=1}^{2} \alpha_{k}(t) \varphi_{k}(t) e^{\tau_{k}}+\frac{\left(H_{1}(t), \chi_{2}(t)\right)}{\lambda_{1}(t)-\lambda_{2}(t)} \varphi_{2}(t) e^{\tau_{1}}+\frac{\left(H_{2}(t), \chi_{1}(t)\right)}{\lambda_{2}(t)-\lambda_{1}(t)} \varphi_{1}(t) e^{\tau_{2}}\right)+ \\
& +\left(\begin{array}{cc}
\sum_{1 \leq|m| \leq N_{z},}, & z^{e_{1}+m}(t) \sigma_{1}+\sum_{1 \leq|m| \leq N_{z},}, \quad z^{e_{1}+m}(t) \sigma_{2} \\
m_{3}+1=m_{4} & m_{4}+1=m_{3}
\end{array}\right) e^{\tau_{1}}+ \\
& +\left(\begin{array}{cc}
\sum_{1 \leq|m| \leq N_{z},}, & z^{e_{2}+m}(t) \sigma_{1}+\sum_{1 \leq|m| \leq N_{z},}, z^{e_{2}+m}(t) \sigma_{2} \\
m_{3}+1=m_{4} & =m_{3}
\end{array}\right) e^{\tau_{2}}+ \\
& +Q_{1}(t) e^{\tau_{1}}+Q_{2}(t) e^{\tau_{2}}, \chi_{k}(t) e^{\tau_{k}}>\equiv 0 \quad \forall t \in\left[t_{0}, T\right], k=1,2 .
\end{aligned}
$$

Performing here scalar multiplication, we obtain linear ordinary differential equations with respect to the functions $\alpha_{k}(t)$, involved in the solution (15) of system (10). Attaching to them the initial conditions 
$\alpha_{k}\left(t_{0}\right)=\frac{1}{2}\left(z_{*}, \chi_{k}\left(t_{0}\right)\right), k=1,2$, computed earlier, we find uniquely the functions $\alpha_{k}(t) \in C^{\infty}\left(\left[t_{0}, T\right], \mathbb{C}^{1}\right)$, $k=1,2$, and, therefore, we construct solution (15) in the space in a unique way. The theorem is proved.

Applying Theorems 1 and 2 to iterative problems $\left(10_{k}\right)$ (in this case, the right-hand sides $H^{(k)}(t, \tau)$ of these problems are embedded in the space $U$, i.e. $H^{(k)}(t, \tau)$ we replace with $\left.\hat{H}^{(k)}(t, \tau) \in U\right)$, we find uniquely their solutions in space $U$ and construct series (7). Just as in [3], we prove the following statement.

Theorem 3. Suppose that conditions (1) - 2) are satisfied for system (2). Then, when $\varepsilon \in\left(0, \varepsilon_{0}\right]\left(\varepsilon_{0}>0\right.$ is sufficiently small), system (2) has a unique solution $z(t, \varepsilon) \in \mathrm{C}^{1}\left([0, T], \mathbb{C}^{2}\right)$; in this case, the estimate

$$
\left\|z(t, \varepsilon)-z_{\varepsilon N}(t)\right\|_{C[0, T]} \leq c_{N} \varepsilon^{N+1},
$$

holds true, where $z_{\varepsilon N}(t)$ is the restriction (for $\tau=\frac{\psi(t)}{\varepsilon}$ ) of the $N$-partial sum of series (9) (with coefficients $z_{k}(t, \tau) \in U$, satisfying the iteration problems $\left.\left(10_{k}\right)\right)$, and the constant $c_{N}>0$ does not depend on $\varepsilon \in\left(0, \varepsilon_{0}\right]$.

\section{Construction of the solution of the first iteration problem in space $U$.}

Using Theorem 1, we will try to find a solution to the first iteration problem $\left(10_{0}\right)$. Since the right side $h(t)$ of the system $\left(10_{0}\right)$ satisfies condition (11), this system has (according to (15)) a solution in space $U$ in the form

$$
z_{0}(t, \tau)=z_{0}^{(0)}(t)+\sum_{k=1}^{2} \alpha_{k}^{(0)}(t) \varphi_{k}(t) e^{\tau_{k}}
$$

where $z_{0}^{(0)}(t)$ is the solution of the integrated system

$$
z_{0}^{(0)}(t)=\int_{t_{0}}^{t}\left(-A^{-1}(t) K(t, s)\right) z_{0}^{(0)}(s) d s-A^{-1}(t) h(t),
$$

$\alpha_{k}^{(0)}(t) \in C^{\infty}\left(\left[t_{0}, T\right], \mathcal{C}^{1}\right)$ are arbitrary functions. Subjecting $(18)$ to the initial condition $z_{0}\left(t_{0}, 0\right)=z^{0}$, we will have

$$
z_{0}^{(0)}\left(t_{0}\right)+\sum_{k=1}^{2} \alpha_{k}^{(0)}\left(t_{0}\right) \varphi_{k}\left(t_{0}\right)=z^{0} \Leftrightarrow \sum_{k=1}^{2} \alpha_{k}^{(0)}\left(t_{0}\right) \varphi_{k}\left(t_{0}\right)=z^{0}+A^{-1}\left(t_{0}\right) h\left(t_{0}\right)
$$

Multiplying this equality scalarly by $\chi_{j}\left(t_{0}\right)$ and taking into account the biorthogonality of the systems $\left\{\varphi_{k}(t)\right\}$ and $\left\{\chi_{j}(t)\right\}$, we find the values $\alpha_{k}^{(0)}\left(t_{0}\right)=\frac{1}{2}\left(z^{0}+A^{-1}\left(t_{0}\right) h\left(t_{0}\right), \chi_{k}\left(t_{0}\right)\right), k=1,2$. To fully compute the functions $\alpha_{k}^{(0)}(t)$, we proceed to the next iteration problem $\left(10_{1}\right)$. Substituting into it the solution (16) of the system $\left(10_{0}\right)$, we arrive at the following system:

$$
\begin{gathered}
L z_{1}(t, \tau)=-\frac{d}{d t} z_{0}^{(0)}(t)-\sum_{k=1}^{2} \frac{d}{d t}\left(\alpha_{k}^{(0)}(t) \varphi_{k}(t)\right) e^{\tau_{k}}+ \\
+\frac{g(t)}{2}\left(e^{\tau_{3}} \sigma_{1}+e^{\tau_{4}} \sigma_{2}\right) B(t)\left(z_{0}^{(0)}(t)+\sum_{k=1}^{2} \alpha_{k}^{(0)}(t) \varphi_{k}(t) e^{\tau_{k}}\right)+ \\
+\sum_{j=1}^{2}\left[\frac{\left(K(t, t) \alpha_{j}^{(0)}(t) \varphi_{j}(t)\right)}{\lambda_{j}(t)} e^{\tau_{j}}-\frac{\left(K\left(t, t_{0}\right) \alpha_{j}^{(0)}\left(t_{0}\right) \varphi_{j}\left(t_{0}\right)\right)}{\lambda_{j}\left(t_{0}\right)}\right]
\end{gathered}
$$

(here we used the expression $\left(6_{1}\right)$ for $R_{1} z(t, \tau)$ and took into account that for $z(t, \tau)=z_{0}(t, \tau)$ only the terms with $e^{\tau_{1}}$ and $e^{\tau_{2}}$ remain in the sum $\left.\left(6_{1}\right)\right)$. It is not difficult to see that the right side

$$
\begin{gathered}
H(t, \tau)=-\frac{d}{d t} z_{0}^{(0)}(t)-\sum_{k=1}^{2} \frac{d}{d t}\left(\alpha_{k}^{(0)}(t) \varphi_{k}(t)\right) e^{\tau_{k}}+ \\
+\frac{g(t)}{2}\left(e^{\tau_{3}} \sigma_{1}+e^{\tau_{4}} \sigma_{2}\right) B(t)\left(z_{0}^{(0)}(t)+\sum_{k=1}^{2} \alpha_{k}^{(0)}(t) \varphi_{k}(t) e^{\tau_{k}}\right)+ \\
+\sum_{j=1}^{2}\left[\frac{\left(K(t, t) \alpha_{j}^{(0)}(t) \varphi_{j}(t)\right)}{\lambda_{j}(t)} e^{\tau_{j}}-\frac{\left(K\left(t, t_{0}\right) \alpha_{j}^{(0)}\left(t_{0}\right) \varphi_{j}\left(t_{0}\right)\right)}{\lambda_{j}\left(t_{0}\right)}\right]
\end{gathered}
$$


of system (20) belongs to space $U$. System (20) is solvable in this space $U$ if and only if conditions (11) are satisfied, which in our case take the form

$$
\begin{aligned}
& \left(-\frac{d}{d t}\left(\alpha_{k}^{(0)}(t) \varphi_{k}(t)\right)+\frac{\left(K(t, t) \alpha_{k}^{(0)}(t) \varphi_{k}(t)\right)}{\lambda_{k}(t)}, \chi_{k}(t)\right)=0 \Leftrightarrow \\
\Leftrightarrow & 2 \frac{d \alpha_{k}^{(0)}(t)}{d t}=\left(\frac{\left(K(t, t) \varphi_{k}(t)\right)}{\lambda_{k}(t)}-\dot{\varphi}_{k}(t), \chi_{k}(t)\right) \alpha_{k}^{(0)}(t), k=1,2 .
\end{aligned}
$$

Attaching to this system the initial conditions $\alpha_{k}^{(0)}\left(t_{0}\right)=\frac{1}{2}\left(z^{0}+A^{-1}\left(t_{0}\right) h\left(t_{0}\right), \chi_{k}\left(t_{0}\right)\right)$, we find uniquely functions

$$
\alpha_{k}^{(0)}(t)=\alpha_{k}^{(0)}\left(t_{0}\right) \exp \left\{\frac{1}{2} \int_{t_{0}}^{t}\left(\frac{\left(K(s, s) \varphi_{k}(s)\right)}{\lambda_{k}(t)}-\dot{\varphi}_{k}(s), \chi_{k}(s)\right) d s\right\}, k=1,2,
$$

therefore, we uniquely calculate the solution (18) of the problem $\left(10_{0}\right)$ in the space $U$. Moreover, the main term of the asymptotic of the solution to problem (2) has the form

$$
\begin{gathered}
z_{\varepsilon 0}(t)=z_{0}^{(0)}(t)+ \\
+\sum_{k=1}^{2} \alpha_{k}^{(0)}\left(t_{0}\right) \exp \left\{\frac{1}{2} \int_{t_{0}}^{t}\left(\frac{\left(K(s, s) \varphi_{k}(s)\right)}{\lambda_{k}(s)}-\dot{\varphi}_{k}(s), \chi_{k}(s)\right) d s\right\} \varphi_{k}(t) e^{\frac{1}{\varepsilon} \int_{t_{0}}^{t} \lambda_{k}(\theta) d \theta},
\end{gathered}
$$

where $\alpha_{k}^{(0)}\left(t_{0}\right)=\frac{1}{2}\left(z^{0}+A^{-1}\left(t_{0}\right) h\left(t_{0}\right), \chi_{k}\left(t_{0}\right)\right), k=1,2, z_{0}^{(0)}(t)$ is the solution of the integra system (19).

This work is supported by the grant № AP05133858 «Contrast structures in singularly perturbed equations and their application in the theory of phase transitions» (2018-2020) by the Committee of Science, Ministry of Education and Science of the Republic of Kazakhstan.

\section{References}

1 Фещенко С.Ф. Асимптотические методы в теории линейных дифференциальных уравнений / С.Ф. Фещенко, Н.И. Шкиль, Л.Д. Николенко. — Киев: Наук. думка, 1966. - 261 с.

2 Далецкий Ю.Л. Асимптотический метод для некоторых дифференциальных уравнений с осциллирующими коэффициентами / Ю.Л. Далецкий // ДАН СССР. - 1962. - 143 - № 5. - С. 1026-1029.

3 Ломов С.А. Введение в общую теорию сингулярных возмущений / С.А. Ломов. - М.: Наука, 1981. $-400 \mathrm{c}$.

4 Ломов С.А. Основы математической теории пограничного слоя / С.А. Ломов, И.С. Ломов. - М.: Изд-во МГУ, 2011. - 456 с.

5 Рыжих А.Д. Асимптотическое решение линейного дифференциального уравнения с быстро осциллирующим коэффициентом / А.Д. Рыжих // Тр. МЭИ. - 1977. - 357. - С. 92-94.

6 Рыжих А.Д. Применение метода регуляризации для уравнения с быстро осциллирующими коэффициентами / А.Д. Рыжих // Материалы Всесоюз. конф. по асимп. методам. - Ч. І. - Алма-Ата: Наука, 1979. - С. 64-66.

7 Иманбаев Н.С. Алгоритм метода регуляризации для сингулярно возмущенной задачи с нестабильным значением ядра интегрального оператора / Н.С. Иманбаев, Б.Т. Калимбетов, М.А. Темирбеков // Вестн. Караганд. ун-та. Сер. Математика. - 2013. - № 4. - С. 64-70.

8 Калимбетов Б.Т. Математическое описание внутреннего пограничного слоя для нелинейной интегродифференциальной системы / Б.Т. Калимбетов, Б.И. Ескараева, М.А. Темирбеков // Вестн. Караганд. ун-та. Сер. Математика. - 2014. - № 3. - 75. - С. 77-87.

9 Ескараева Б.И. Дискретный пограничный слой в случае нулевых точек спектра для систем интегродифференциальных уравнений / Б.И. Ескараева, Б.Т. Калимбетов, М.А. Темирбеков // Вестн. Караганд. ун-та. Сер. Математика. - 2014. - № 3. - 75. - С. 88-95.

10 Kalimbetov B.T. Internal boundary layer for integral-differential equations with zero spectrum of the limit operator and rapidly changing kernel / B.T. Kalimbetov, B.I. Yeskarayeva, A.S. Tolep // Applied Mathematical Sciences. - 2015. - 9. - 141-144. - P. 7149-7165. 
11 Kalimbetov B.T. Asymptotic solution of singular perturbed problems with an instable spectrum of the limiting operator / B.T. Kalimbetov, M.A. Temirbekov, Zh.O. Habibullaev // Abstract and Applied Analysis. - 2012. - Vol. 2012, No. 120192. - 16 p.

12 Kalimbetov B.T. Regularized asymptotical solutions of integro-differential systems with spectral singularites / B.T. Kalimbetov, N.S. Imanbaev, D.A. Sapakov, L.T. Tashimov // Advances in Difference Equations. - 2013. - Vol. 109. DOI: 10.1186/1687-1847-2013-109.

13 Safonov V.F. A regularization method for systems with instable spectral of the kernel of the integral operator / V.F. Safonov, B.T. Kalimbetov // Differential Equations. - 31. - 4. - 1995. - P. 647-656.

14 Safonov V.F. Regularized asymptotic solutions of the initial problem of systems of integro-partial differential equations / V.F. Safonov, A.A. Bobodzhanov // Mathematical Notes. - 2017. - 102. - 1. P. 22-30.

15 Safonov V.F. Regularized asymptotics of solutions to integro-differential partial differential equations with rapidly varying kernels / V.F. Safonov, A.A. Bobodzhanov // Ufimsk. Mat. Zh. - 2018. - 10. - 2. - P. 3-12.

16 Bobodzhanov A.A. A generalization of the regularization method to the singularly perturbed integrodifferential equations with partial derivatives / A.A. Bobodzhanov, V.F. Safonov // Russian Math. (Iz. VUZ). - 2018. - 62. - 3. - P. 6-17.

17 Bobodzhanov A.A. A problem with inverse time for a singularly perturbed integro-differential equation with diagonal degeneration of the kernel of high order / A.A. Bobodzhanov, V.F. Safonov // Izv. Math. - 2016. - 80. - 2. - P. 285-298.

18 Bobodzhanov A.A. Asymptotic solutions of Fredholm integro-differential equations with rapidly changing kernels and irreversible limit operator / A.A. Bobodzhanov, V.F. Safonov // Russian Math. (Iz. VUZ). - 2015. - 59. - 10. - P. 1-15.

19 Bobodzhanov A.A. The method of normal forms for singularly perturbed systems of Fredholm integrodifferential equations with rapidly varying kernels / A.A. Bobodzhanov, V.F. Safonov // Sb. Math. 2013. - 204. - 7. - P. 979-1002.

20 Safonov V.F. Volterra integral equations with rapidly varying kernels and their asymptotic integration / V.F. Safonov, A.A. Bobodzhanov // Sb. Math. - 2001. - 192. - 8. - P. 1139-1164.

21 Safonov V.F. «Splashes» in Fredholm integro-differential equations with rapidly varying kernels / V.F. Safonov, A.A. Bobodzhanov // Math. Notes. - 2009. - 85. - 2. - P. 153-167.

22 Сафонов В.Ф. Курс высшей математики. Сингулярно возмущенные задачи и метод регуляризации / В.Ф. Сафонов, А.А. Бободжанов. - М.: Издательский дом МЭИ, 2012. - 414 с.

\author{
Б.Т. Калимбетов, В.Ф. Сафонов
}

\title{
Жылдам осцилляцияланатын коэффициентті сингуляр ауытқыған интегро-дифференциалдық теңдеулер
}

\begin{abstract}
Динамикалық орнықтылықпен, периодты құрылымға ие ортамен байланысты және басқа да қолданбалы мәселелерді зерттеулер жылдам осцилляцияланатын коэффициентті дифференциалдық теңдеулермен айналысуды қажет етеді. Мұндай коэффициентті дифференциалдық теңдеулер жүйелерінің асимптотикалық интегралдау бөлшектеу және регуляризация әдістермен жүргізілген. Осы жұмыста интегралды-дифференциалдық теңдеулер жүйесі қарастырылған. Зерттеудің негізгі мақсаты - интегралдық мүшенің алғашқы есептің шешімінің асимптотикасына әсерін зерттеу. Есепте резонанс болмаған жағдай, яғни жылдам осцилляцияланатын коэффициенттің бүтін сызықтық комбинациясының жиіліктері шекті оператордың спектрінің жиілігімен сәйкес келмейтін жағдай, зерттелген.
\end{abstract}

Kiлm сөздер: сингуляр ауытқу, интегро-дифференциалдық теңдеу, жылдам осцилляцияланатын коэффициент, регуляризация, асимптотикалық жинақтылық. 
Б.Т. Калимбетов, В.Ф. Сафонов

\title{
Интегро-дифференциальные сингулярно возмущенные уравнения с быстро осциллирующими коэффициентами
}

\begin{abstract}
При исследовании различных вопросов, связанных с динамической устойчивостью, со свойствами сред с периодической структурой, при исследовании других прикладных задач приходится иметь дело с дифференциальными уравнениями с быстро осциллирующими коэффициентами. Асимптотическое интегрирование дифференциальных систем уравнений с такими коэффициентами проводилось методами расщепления и регуляризации. В настоящей работе рассмотрена система интегральнодифференциальных уравнений. Основная цель исследования состоит в выявлении влияния интегрального члена на асимптотику решения исходной задачи. Изучен случай отсутствия резонанса, т.е. случай, когда целочисленная линейная комбинация частот быстро осциллирующего коэффициента не совпадает с частотой спектра предельного оператора.
\end{abstract}

Ключевые слова: сингулярное возмущение, интегрально-дифференциальное уравнение, быстро осциллирующий коэффициент, регуляризация, асимптотическая сходимость.

\section{References}

1 Feschenko, S.F., Shkil, N.I., \& Nikolenko, L.D. (1966). Asimptoticheskie metody v teorii lineinykh differentsialnykh uravnenii [Asymptotic methods in the theory of linear differential equations]. Kiev: Naukova Dumka [in Russian].

2 Daletsky, Yu.L. (1962). Asimptoticheskii metod dlia nekotorykh differentsialnykh uravnenii s ostsilliruiushchimi koeffitsientami [The asymptotic method for some differential equations with oscillating coefficients]. Doklady Akademii Nauk SSSR - Reports of the Academy of Science of the USSR, 143, 5, 1026-1029 [in Russian].

3 Lomov, S.A. (1981). Vvedenie v obshchuiu teoriiu sinhuliarnykh vozmushchenii [Introduction to the general theory of singular perturbations]. Moscow: Nauka [in Russian].

4 Lomov, S.A., \& Lomov, I.S. (2011). Osnovy matematicheskoi teorii pohranichnoho sloia [Foundations of mathemathical theory of boundary layer]. Moscow: Izdatelstvo MHU [in Russian].

5 Ryzhih, A.D. (1977). Asimptoticheskoe reshenie lineinoho differentsialnoho uravneniia s bystro ostsilliruiushchim koeffitsientom [Asymptotic solution of a linear differential equation with a rapidly oscillating coefficient]. Trudy MEI - The works of MEI, 357, 92-94 [in Russian].

6 Ryzhih, A.D. (1979). Primenenie metoda rehuliarizatsii dlia uravneniia s bystro ostsilliruiushchimi koeffitsientami [Application of the regularization method for an equation with rapidly oscillating coefficients]. Materialy Vsesoiuznoi konferentsii po asimp. metodam - Mater. All-union. conf. by asymp. methods, Part I. Alma-Ata, Science, 64-66 [in Russian].

7 Imanbaev, N.S., Kalimbetov, B.T., \& Temirbekov, M.A. (2013). Alhoritm metoda rehuliarizatsii dlia sinhuliarno vozmushchennoi zadachi s nestabilnym znacheniem yadra intehralnoho operatora [Algorithm of the regularization method for a singularly perturbed problem with unstable value of kernel of the integral operator]. Vestnik Karahandinskoho universiteta. Seriia Matematika - Bulletin of the Karaganda University. Mathematics series, No. 4, 64-70 [in Russian].

8 Kalimbetov, B.T., Yeskaraeva, B.I., \& Temirbekov, M.A. (2014). Matematicheskoe opisanie vnutrenneho pohranichnoho sloia dlia nelineinoi intehro-differentsialnoi sistemy [Mathematical description of the inner boundary layer for a nonlinear integro-differential system]. Vestnik Karahandinskoho universiteta. Seriia Matematika - Bulletin of the Karaganda University. Mathematics series, 75, No. 3, 77-87 [in Russian].

9 Yeskaraeva, B.I., Kalimbetov, B.T., \& Temirbekov, M.A. (2014). Diskretnyi pohranichnyi sloi v sluchae nulevykh tochek spektra dlia sistem intehro-differentsialnykh uravnenii [Discrete boundary layer in the case of zero points of the spectrum for systems of integro-differential equations]. Vestnik Karahandinskoho universiteta. Seriia Matematika - Bulletin of the Karaganda University. Mathematics series, 75, No. 3, 88-95 [in Russian]. 
10 Kalimbetov, B.T., Yeskarayeva, B.I., \& Tolep A.S. (2015). Internal boundary layer for integral-differential equations with zero spectrum of the limit operator and rapidly changing kernel. Journal Applied Mathematical Sciences, 9, 141-144, 7149-7165.

11 Kalimbetov, B.T., Temirbekov, M.A., \& Habibullaev, Zh.O. (2012). Asymptotic solution of singular perturbed problems with an instable spectrum of the limiting operator. Journal Abstract and Applied Analysis, Vol. 2012, No. 120192, 16.

12 Kalimbetov, B.T., Imanbaev, N.S., Sapakov, D.A., \& Tashimov, L.T. (2013). Regularized asymptotical solutions of integro-differential systems with spectral singularites. Journal Advances in Difference Equations, 109. DOI: 10.1186/1687-1847-2013-109.

13 Safonov, V.F., \& Kalimbetov, B.T. (1995). A regularization method for systems with unstable spectral value of the kernel of the integral operator. Journal Differential Equations, 31(4), 647-656.

14 Safonov, V.F., \& Bobodzhanov, A.A. (2017). Regularized asymptotic solutions of the initial problem of systems of integro-partial differential equations. Mathematical Notes, 102, 1, 22-30.

15 Safonov, V.F., \& Bobodzhanov, A.A. (2018). Regularized asymptotics of solutions to integro-differential partial differential equations with rapidly varying kernels. Ufimsk. Mat. Zh., 10, 2, 3-12.

16 Bobodzhanov, A.A., \& Safonov, V.F. (2018). A generalization of the regularization method to the singularly perturbed integro-differential equations with partial derivatives. Journal Russian Math. (Izvest. VUZ), 62, 3, 6-17.

17 Bobodzhanov, A.A., \& Safonov, V.F. (2016). A problem with inverse time for a singularly perturbed integro-differential equation with diagonal - of the kernel of high order. Journal Izvest. Math, 80, 2, $285-298$.

18 Bobodzhanov, A.A., Safonov, V.F. (2015). Asymptotic solutions of Fredholm integro-differential equations with rapidly changing kernels and irreversible limit operator. Journal Russian Math. (Izvest. VUZ), 59, 10, 1-15.

19 Bobodzhanov, A.A., \& Safonov, V.F. (2013). The method of normal forms for singularly perturbed systems of Fredholm integro-differential equations with rapidly varying kernels. Sibir. Math J., 204, 7, 979-1002.

20 Safonov, V.F., \& Bobodzhanov, A.A. (2001). Volterra integral equations with rapidly varying kernels and their asymptotic integration. Sibir. Math. J., 192, 8, 1139-1164.

21 Safonov, V.F., \& Bobodzhanov, A.A. (2009). «Splashes» in Fredholm integro-differential equations with rapidly varying kernels. Math. Notes, 85, 2, 153-167.

22 Safonov V.F., Bobodzhanov A.A. (2012). Kurs vysshei matematiki. Sinhuliarno vozmushchennye zadachi $i$ metod rehuliarizatsii [The course of higher mathematics. Singularly perturbed problems and regularization method]. Moscow: Izdatelskii dom MEI [in Russian]. 